\title{
An alternative view at the zero-bias anomaly of metallic point contacts
}

\author{
Kurt Gloos \\ Wihuri Physical Laboratory, Department of Physics and Astronomy, University of Turku, FIN-20014 Turku, Finland \\ Turku University Centre for Materials and Surfaces (MatSurf), FIN-20014 Turku, Finland \\ E-mail:kgloos@utu.fi
}

Received July 1, 2009, revised August 13, 2009

\begin{abstract}
We have studied the zero-bias anomaly of point contacts as function of contact size for a wide range of materials from simple normal metals, superconductors, and magnets to highly correlated heavy-fermion compounds. The size $\delta R$ of the zero-bias anomaly varied in a universal manner proportional to the square of the contact resistance $R$ between $1 \Omega$ and $10 \mathrm{k} \Omega$, that is for contact radii from about $15 \mathrm{~nm}$ down to $0.2 \mathrm{~nm}$. Magnetic impurities, two-level systems, or a tunneling barrier are unlikely to be the main source of this anomaly. We suggest instead Kondo-type scattering of the conduction electrons due to a spontaneous electron spin polarization at the point contact.
\end{abstract}

PACS: 73.20.-r Electron states at surfaces and interfaces;

73.23.-b Electronic transport in mesoscopic systems;

73.63.-b Electronic transport in nanoscale materials and structures;

73.40.Jn Metal-to-metal contacts.

Keywords: point contacts, zero-bias anomaly, Kondo effect, spontaneous polarization.

\section{Introduction}

In 1974 Igor K. Yanson [1] invented point-contact spectroscopy by directly measuring the electron-phonon interaction in metals. However, it was found that very often an additional anomaly accompanies the electron-phonon type spectral features, a maximum or a minimum of the differential contact resistance at zero bias. Till today this so-called zero-bias anomaly can not be easily explained using known mechanisms [2]. The zero-bias anomaly is not very reproducible: It is not always present, its size and width vary even for contacts of the same materials, making its investigation difficult. Two different explanations for the zero-bias anomaly are discussed in the literature: $i)$ Magnetic impurities that cause the wellknown Kondo effect [3-6]. ii) A two-level system, possibly due to lattice distortions created by the contact itself, that resonantly scatters low-energy electrons [7-10]. iii) One could add as third possibility a tunneling barrier which becomes transparent at large bias voltages. For specific point contacts one of those explanations might indeed be valid, but in general this is not the case. Naidyuk and Yanson [2] provide a detailed overview of the problem.
A point contact between two identical normal metals has a resistance of $R(T) \approx 2 R_{K} /\left(a k_{F}\right)^{2}+\rho(T) /(2 a)$, where $R_{K}=h / e^{2}$ is the von Klitzing resistance, $k_{F}$ is the Fermi wavenumber, and $a$ is the contact radius $[2,11]$. It depends on temperature $T$ via the specific resistivity $\rho(T)$. In the ballistic limit electrons cross the contact region on straight trajectories, and the first term dominates. With increasing scattering the second term grows and the contact becomes diffusive or thermal, depending on whether scattering is elastic or inelastic. The above idealized equation for the resistance neglects, among others, a possible interface (tunneling) barrier, reflection due to Fermi velocity mismatch at contacts between different metals, or deviations from a circular contact area, see for example $[2,12,13]$ and references therein. The Drude-Sommerfeld theory of metals predicts [14] a constant product of electrical resistivity and electron mean free path $\rho l=\left(3 \pi R_{K}\right) /\left(2 k_{F}^{2}\right)$, and the temperature dependence of the resistance can be replaced by the energy or bias-voltage dependence of the differential resistance at low temperature

$$
\frac{d V}{d I}(V) \approx \frac{2 R_{K}}{\left(a k_{F}\right)^{2}}\left(1+\frac{a}{l(e V)}\right)
$$


to extract $l(\mathrm{eV})$. Independent scattering processes can be separated according to Matthiessen's rule $1 / l=1 / l_{0}+1 / l_{1}+1 / l_{2}+\ldots$ when they have different energy dependencies of their respective $l_{0}, l_{1}, l_{2}, \ldots$. This is the case for electron-phonon scattering, which sets in at energies above 5-10 meV, depending on the Debye energy, and Kondo scattering at magnetic impurities that is efficient only at small energies. Such an impurity polarizes the surrounding electron spins, forming a polarization cloud with an extension of about $\xi_{K} \approx \hbar v_{F} / k_{B} T_{K}$ and a correspondingly large scattering cross-section. Here $v_{F}$ is the Fermi velocity. The electrical resistivity due to scattering of electrons at Kondo impurities [15]

$$
\Delta \rho(T)=\frac{\Delta \rho_{0}}{2}\left[1-\frac{\ln \left(T / T_{K}\right)}{\sqrt{\ln ^{2}\left(T / T_{K}\right)+S(S+1) \pi^{2}}}\right]
$$

depends on the Kondo temperature $T_{K}$ and the effective spin $S$. It reaches a maximum of $\Delta \rho_{0}=c 2 R_{K} / k_{F}$ at low temperatures that equals the impurity concentration $c$ (impurity per conduction electron) times the resistivity in the so-called unitary limit with ideal retro-reflection. Since a point contact is very small, even a single magnetic impurity can change dramatically the resistance. The contact itself can squeeze the Kondo cloud, thereby enhancing the Kondo temperature $[5,6]$.

\section{Experimental}

We have studied the zero-bias anomaly of point contacts with a wide variety of metals. We found a systematic variation of the size of the anomaly as function of the contact radius, independent of the metals used as electrodes. This observation discards the above mentioned three mechanisms and calls for a new and more generally valid explanation. Our experimental data are based on spear-anvil type point contacts measured at $4.2 \mathrm{~K}$ in liquid helium as well as mechanically controllable break junctions and spear-anvil type contacts measured down to $0.1 \mathrm{~K}$. The latter data are from earlier experiments where those contacts with noticeable zero-bias anomaly were usually discarded. $\mathrm{Cu}$ is a simple normal metal, Ta is a BCS-type superconductor with $T_{c}=4.2 \mathrm{~K}$ (in the experiments described here it is in the normal state), $\mathrm{Nb}$ is a BCS-type superconductor with $T_{c}=9.1 \mathrm{~K}$, and $\mathrm{Ni}$ is a band-ferromagnet with $T_{\text {Curie }}=627 \mathrm{~K}$ [14]. $\mathrm{CeAl}_{2}$ is a heavy-fermion antiferromagnet with $T_{\text {Neel }}=3.8 \mathrm{~K}$, see Ref. 16 for further information on material properties and break-junction experiments. AuIn 2 is a BCS-type superconductor with $T_{c}=0.21 \mathrm{~K}$. Its contacts with $\mathrm{Cu}$ have been reported in [17]. From the bulk resistivity at $4.2 \mathrm{~K}$ (above $9.1 \mathrm{~K}$ for $\mathrm{Nb}$ ) we estimate electron mean free paths of at least $10 \mathrm{~nm}$ for all samples. That means contacts down to about $1 \Omega$ should be in the ballistic limit.

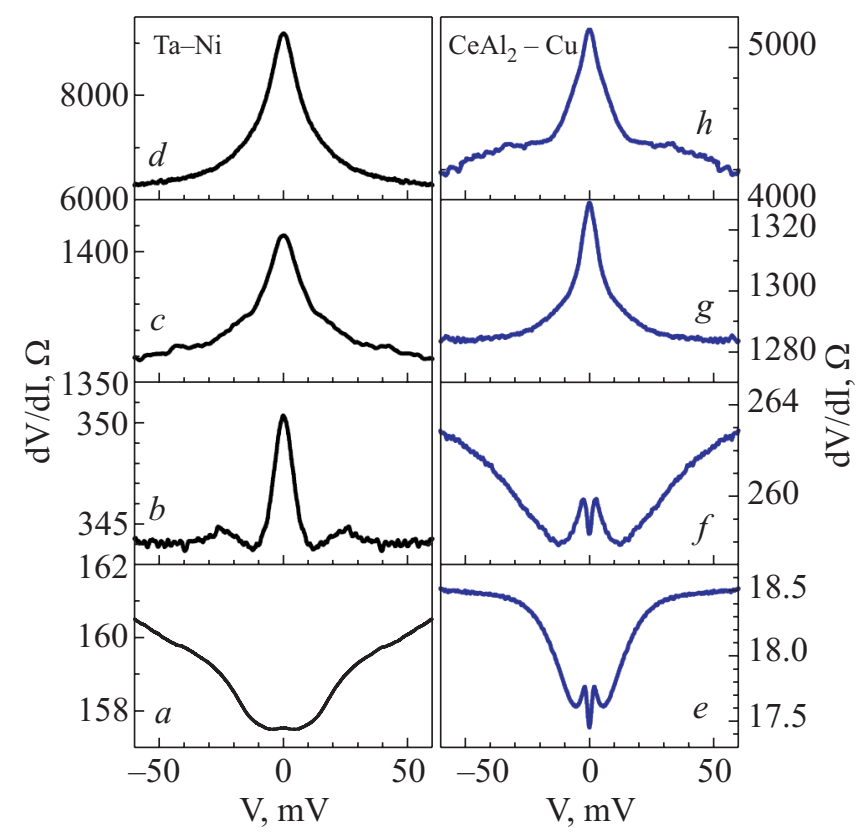

Fig. 1. Typical $d V / d I(V)$ spectra of a Ta-Ni (left panel) and $\mathrm{CeAl}_{2}-\mathrm{Cu}$ (right panel) contacts at $4.2 \mathrm{~K}$.

Figure 1 displays a series of typical spectra of $\mathrm{Ta}-\mathrm{Ni}$ and $\mathrm{CeAl}_{2}-\mathrm{Cu}$ contacts. Low-resistance contacts usually showed the zero-bias anomaly, unless it was below the noise level, together with the typical features of electron-phonon interaction as the Ta-Ni contact in Fig. 1, $a$ indicating ballistic transport. Low-resistance $\mathrm{CeAl}_{2}$ contacts had a minimum at zero bias inside a maximum. We attribute this feature to the onset of antiferromagnetic ordering in this compound because it is also present at $\mathrm{CeAl}_{2}$ break junctions at very low temperatures [16]. Similarly, low-resistance contacts with $\mathrm{Nb}$ showed the BTK double-minimum structure [18] of Andreev reflection. When the contact radius was reduced and the resistance increased, the relative magnitude of the zero-bias anomaly grew and at the same time the spectroscopic features of electron-phonon interaction, magnetism, and superconductivity became suppressed. Andreev-reflection anomalies of contacts with $\mathrm{Nb}$ vanished completely, while those of contacts with $\mathrm{AuIn}_{2}$ coexisted with the zero-bias anomaly, possibly due to the very large superconducting coherence length of this compound [17]. In general, the zero-bias anomaly appeared to be more robust than the structure due to electron-phonon scattering. In rare cases (one out of a hundred contacts, except $\mathrm{Cu}-\mathrm{Cu}$ contacts) we found an inverted zero-bias anomaly, that is a minimum, of comparable size as the maximum. Its origin is unclear - possibly due to an accidental fabrication of a tunnel junction - and because it was only seldomly observed we could not study it in detail. $\mathrm{Cu}-\mathrm{Cu}$ contacts had zero-bias minima as well as maxima, with 


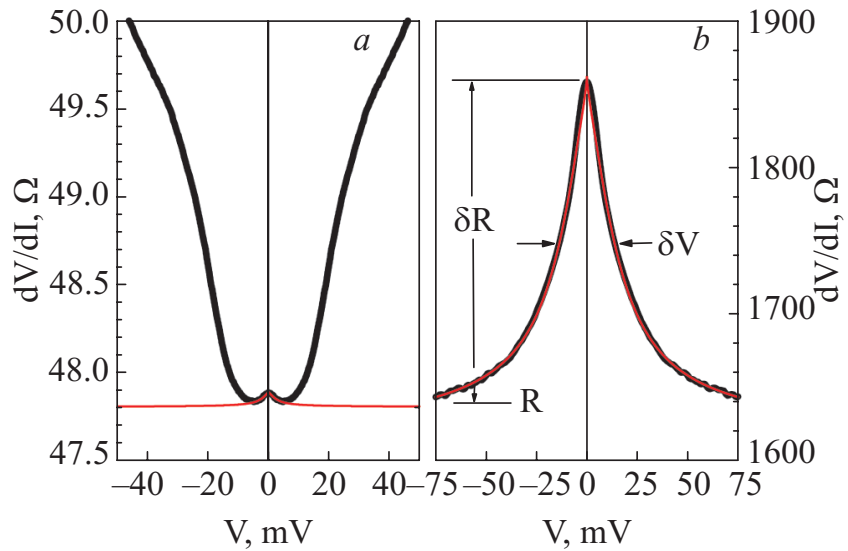

Fig. 2. Differential resistance of two typical $\mathrm{Cu}-\mathrm{Ni}$ contacts at $4.2 \mathrm{~K}$ (thick solid lines). Contact $(a)$ has a rather small zerobias anomaly, but one can clearly see the rise of the differential resistance at about $20 \mathrm{mV}$ due to electron-phonon interaction. Contact $(b)$ has no resolvable electron-phonon interaction, but a huge zero-bias anomaly. The definition of $R, \delta R$, and $\delta V$ is shown. The zero-bias anomalies are fitted using Eqs. (1) and (2) with $S=0.2$ (thin solid lines). The Kondo voltage is (a) $2.5 \mathrm{mV}$ and (b) $16 \mathrm{mV}$, respectively.

minima appearing preferrably at low $R$ and maxima at large $R$ contacts.

Figure 2 shows two typical spectra of $\mathrm{Cu}-\mathrm{Ni}$ contacts. The differential resistance increases logarithmically towards zero bias as expected for Kondo scattering Eq. (2). With this interpretation one can directly read off the Kondo voltage $V_{K}=\delta V / 2$, and thus the Kondo temperature $T_{K}$, as half width of the zero-bias anomaly. However, the effective spin $S \approx 0.2$ was always smaller than the expected $S=0.5$. Figure 3 shows that over a wide range of contact resistances $\delta R \sim R^{2}$. (Note that the Ni-Ni as well as the $\mathrm{Cu}-\mathrm{Cu}$ homocontacts seem to have a systematically weaker dependence. For the $\mathrm{Cu}-\mathrm{Cu}$ contacts the exponent of the power law could be as low as $3 / 2$ instead of 2.) Here $R$ is the (extrapolated) zero-bias resistance without zero-bias anomaly and $\delta R$ the additional resistance of the anomaly. The observed dependence contrasts the typical spectroscopic features like the electron-phonon interaction which vary as $\delta R \sim \sqrt{R}$ according to Eq. (1). This square-root dependence is valid when the mean free path for the specific scattering mechanism depends only on energy (and not on the specific contact), and corrections due to backscattering are small, so that $R$ is a good approximation for the ballistic part $2 R_{K} /\left(a k_{F}\right)^{2}$ of the contact resistance. The behavior does not change between small and large contacts, indicating that the barely resolved tiny zero-bias anomalies at small $R$ develop directly into the huge anomalies at large $R$. Thus the same mechanism is responsible for those anomalies, independent of the con- tact size. Within the huge scattering of data points in Fig. 4, one can also recognize a trend towards larger widths when the resistance increases.

\section{Discussion}

First of all, we can not exclude the Kondo effect, two-level systems, or a tunneling barrier, or a combination of all three, being present at specific contacts. In principle, one can always reconstruct the zero-bias anomalies using specific assumptions, like the type of magnetic impurities and their concentration. However, the systematic variation of the size of the zero-bias anomaly with contact resistance would pose rather strong constraints on those mechanisms: One would have to assume a certain type of impurities as well as a certain variation of their density with contact resistance. Or the width and height of the tunneling barrier would have to vary in a specific way. In addition, those mechanisms (impurity scattering or tunneling) would have to be the same for all investigated metals. This can be savely discarded. In the case of $\mathrm{AuIn}_{2}-\mathrm{Cu}$ contacts [17] or the contacts with $\mathrm{Nb}$ a tunneling barrier can be immediately excluded as explanation for the ZBA anomalies, because it does not show up in the Andreev reflection spectra. On the contrary Andreev reflection indicates a negligible tunnel barrier with a small BTK-theory $Z$ parameter [18] of less than about 0.5 , independent of the contact resistance.

We use Eq. (1) to estimate the electron mean free path as represented by the zero-bias anomaly. Ballistic contacts have a radius $a \approx 15 \mathrm{~nm} / \sqrt{R(\Omega)}$, assuming a typical Fermi wave number $k_{F}=15 \mathrm{~nm}^{-1}$ of metals [14]. At large voltages the zero-bias anomaly is suppressed and therefore $l_{0} \approx a R / \delta R \sim a^{3}$ is the scattering length at zero bias. At large contacts $l_{0}$ becomes very large, while at atomic size contacts it is comparable to the contact radius as if the contact itself was the «impurity». The origin of this electron scattering could be magnetic impurities with effective spin $S \approx 0.2$ as indicated by the shape of the spectra (Fig. 2). Deviations from the ideal Eq. (2) might be attributed to local heating due to a short electron mean free path near the contact or the finite measuring temperature.

One way to describe the effects of such a hypothetical impurity is the so-called unitary limit of Eq. (2). With the impurity concentration $c$ the zero-bias resistance should increase by $\delta R=c \sqrt{2 R_{K} R}$, in clear contradiction with the experimental results in Fig. 3. To fit the data would require to vary the impurity concentration from contact to contact, as already mentioned above. The width of the anomalies in Fig. 4 is typically around $10 \mathrm{mV}$, corresponding to Kondo temperatures of around $100 \mathrm{~K}$ and Kondo lengths of around $0.1 \mu \mathrm{m}$. The parameter which should determine the size of the Kondo cloud and which we can not directly access is the opening angle of the con- 


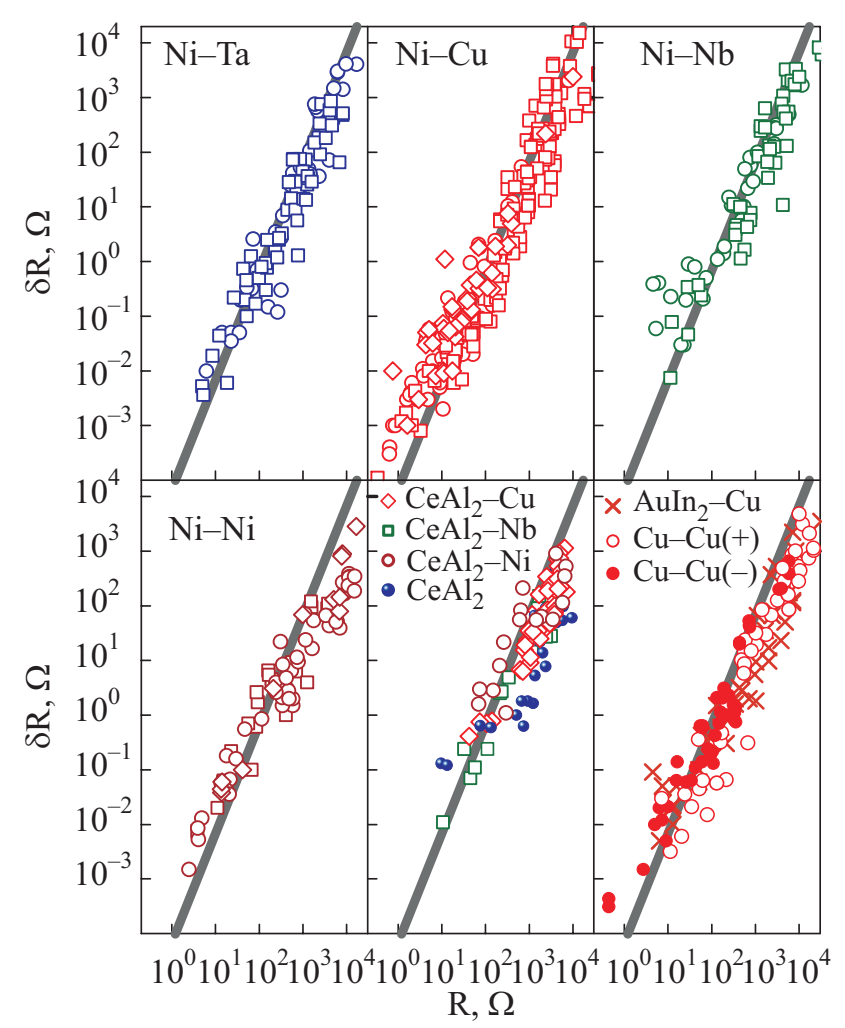

Fig. 3. Magnitude $\delta R$ of the zero-bias anomaly as function of resistance $R$ for the indicated metal contacts. The spear-anvil type contacts have the two metals shown (like Ni-Ta). Break junctions are denoted by a single metal $\left(\mathrm{CeAl}_{2}\right)$. For the $\mathrm{Cu}-\mathrm{Cu}$ contacts we show the magnitude of the maxima (open symbols) as well as the minima (closed symbols). The measuring temperature was $4.2 \mathrm{~K}$, except $\mathrm{CeAl}_{2}$ break junctions $(0.1 \mathrm{~K})$ and $\mathrm{AuIn}_{2}-\mathrm{Cu}$ point contacts $(0.3 \mathrm{~K})$. The thick solid lines are $\delta R=(9 \pi / 16) R^{2} / R_{K}$ as discussed in the text. Different symbols for the $\mathrm{Ni}-(\mathrm{Ta}, \mathrm{Cu}, \mathrm{Nb}, \mathrm{Ni})$ data indicate different series of measurements, for example where the tip was freshly cut. No such distinction is made for the other contacts.

tact. A short flat contact could have a large $\xi_{K}$, and a contact like a short wire a correspondingly small $\xi_{K}$. The estimated $0.1 \mu \mathrm{m}$ is much larger than the contact radius, meaning that it is unlikely to find more than one of those impurities in the contact volume. We estimate the number of impurities that contribute to the zero-bias anomaly as follows: A contact volume $v_{C} \approx 4 \pi a^{3} / 3$ contributes to the resistance through backscattering, and there are $c$ impurities per conduction electron. With the electron density $n=k_{F}^{3} / 3 \pi^{2}$ the number of impurities at the contact is therefore $N=c n v_{C}$. According to the Hamann formula Eq. (2) these impurities contribute up to $\delta R \approx c R_{K} / a k_{F}$ to the contact resistance at low temperatures, assuming ideal retro-reflection. Since we identify $R$ with the ballistic part $2 R_{K} /\left(a k_{F}\right)^{2}$, we can replace both the concentration and the contact radius to get $N \approx(16 / 9 \pi) R_{K} \delta R / R^{2}$, which depends only on the size $\delta R$ of the zero-bias ano-

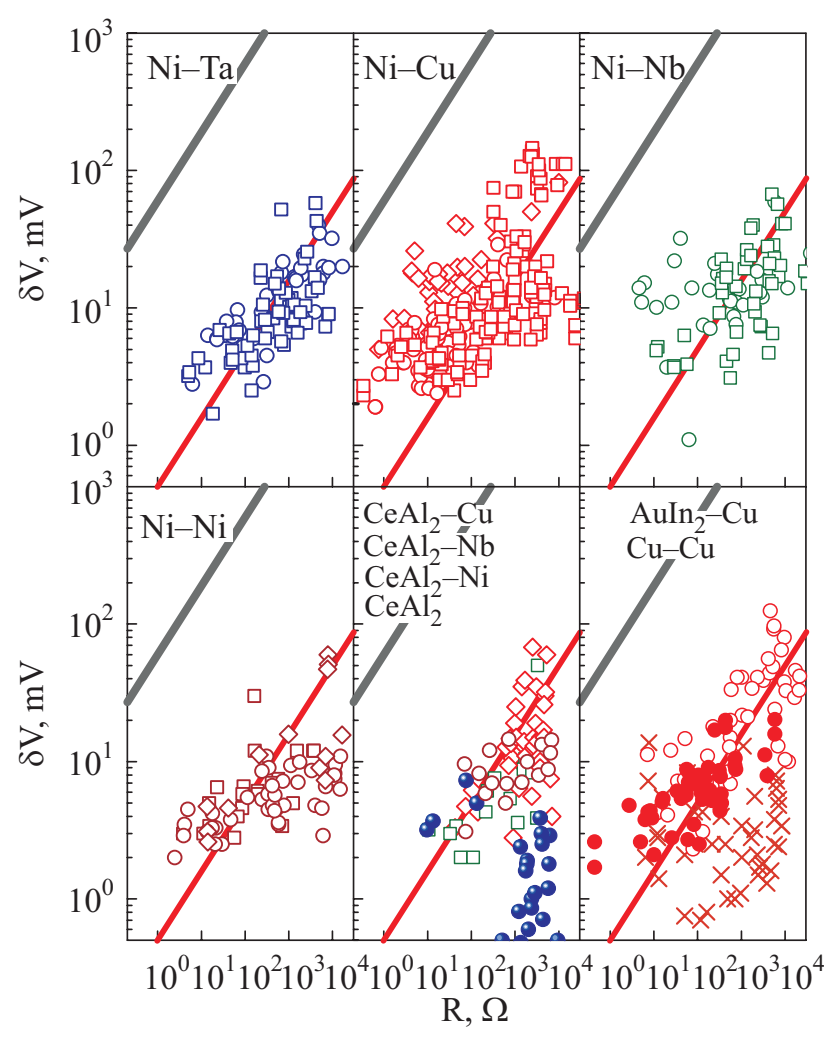

Fig. 4. Full width at half maximum $\delta V$ of the zero-bias anomaly as function of resistance $R$ for the indicated contacts. The same symbols as in Fig. 3 are used. The thick solid lines are $\delta V=2 \hbar v_{F} / e a$ when the Kondo length equals the contact radius. The thin solid lines through the data points represent a 100-times larger Kondo length $\xi_{K}=100 a$.

maly and the contact resistance $R$. The magnitude of the zero-bias anomaly for a single impurity $(N=1)$

$$
\delta R=\frac{9 \pi}{16} \frac{R^{2}}{R_{K}}
$$

describes the experimental data in Fig. 3 quite well. It is consistent with what we have deduced from the width of the anomaly. This leads us to believe that indeed a single impurity determines the zero-bias anomalies.

On a small scale, pronounced Kondo phenomena were observed, for example, for tunneling into a single magnetic atom on a metallic surface [19]. But magnetic impurities are not really needed. Electrons also have a spin and therefore spin-spin scattering should be possible, and lead to the Kondo effect if the number of participating electrons is small. This has been observed in quantum-point contacts and quantum dots in a two-dimensional electron gas, where a single electron sits either in the dot [20-23] or in a shallow potential minimum near the center of the point contact [24]. Transport through the constriction depends then on the spin of this single electron. We suggest here a similar picture that one or few electrons are trapped near the contact and polarize the 
conduction electrons in the contact region. This polarization cloud does not sit in the center of the contact, but on one or on both sides in the electrodes. Indeed, some of our contacts had a zero-bias anomaly apparently composed of two different parts, as if each electrode had its own polarization cloud with different extension. One might further speculate that if two clouds are coupled well enough through the contact that the spins show in the same direction, electrons would flow through the contact region with reduced scattering. This would offer a rather straightforward explanation for the observed zero-bias minima. An alternative explanation might be scattering at a spin glass like state as observed at some point contacts with magnetic impurities [25].

A rather simple explanation for electron trapping at a 3D metallic contact is derived from the boundary condition of electron wave functions which must have nodes at the metal surface. This corresponds to a reduced electron density there which extends also to the contact, and thus to a positive net charge which can attract electrons [26]. It should be more pronounced the smaller the contact is with respect to the electron wavelength, that is big effects are to be expected at atomic-size contacts, similar to what we have observed.

Another scenario has been suggested recently for atomic contacts, that is contacts with few conducting channels and resistances near $R=h / 2 e^{2} \approx 13 \mathrm{k} \Omega$, of the ferromagnets $\mathrm{Fe}, \mathrm{Co}$, and $\mathrm{Ni}$ that showed spectra with Fano-type anomalies [27]. These anomalies were attributed to the Kondo effect caused by the changed band structure at the contact. Whether this could play a role in our experiments is unclear.

\section{Conclusion}

We have found a surprisingly reproducible dependence of the magnitude of the zero-bias anomalies as function of the size of the contacts for a large number of different type of metals. This calls for a common explanation. Since these anomalies can be described by Kondo scattering at a single magnetic impurity in the unitary limit, we suggest a spontaneous electron spin polarization at the contact interface. This would have serious consequences for the interpretation of a number of point-contact experiments, for example those that use Andreev reflection to measure the surface polarization of magnetic samples.
1. I.K. Yanson, Sov. Phys. JETP 39, 506 (1974).

2. Yu.G. Naidyuk and I.K. Yanson, Point-Contact Spectroscopy, Springer Science+Business Media Inc. (2005), p. 99.

3. A.G.M. Jansen, A.P. van Gelder, P. Wyder, and S. Strässler, J. Phys. F: Metal Phys. 11, L15 (1981).

4. Yu.G. Naidyuk, O.I. Shklyarevskii, and I.K. Yanson, Fiz. Nizk. Temp. 8, 725 (1982) [Sov. J. Low Temp. Phys. 8, 362 (1982).

5. I.K. Yanson, V.V. Fisun, R. Hesper, A.V. Khotkevich, J.M. Krans, J.A. Mydosh, and J.M. van Ruitenbeek, Phys. Rev. Lett. 74, 302 (1995).

6. N. van der Post, F.L. Mettes, J.A. Mydosh, J.M. van Ruitenbeek, and I.K. Yanson, Phys. Rev. B53, R476 (1996); N. van der Post, J.A. Mydosh, J.M. van Ruitenbeek, and I.K. Yanson, Phys. Rev. B53, 15106 (1996).

7. D.C. Ralph and R.A. Buhrman, Phys. Rev. Lett. 69, 2118 (1992).

8. R.J.P. Keijsers, O.I. Shklyarevskii, and H. van Kempen, Phys. Rev. B51, 5628 (1995).

9. R.J. P. Keijsers, O.I. Shklyarevskii, and H. van Kempen, Phys. Rev. Lett. 77, 3411 (1996).

10. J. Kozub, Sov. Phys. Solid State 26, 1186 (1984).

11. A.G.M. Jansen, A.P. van Gelder, and P. Wyder, J. Phys. C13, 6073 (1980).

12. K. Gloos, F.B. Anders, B. Buschinger, C. Geibel, K. Heuser, F. Jährling, J.S. Kim, R. Klemens, R. Müller-Reisener, C. Schank, and G.R. Stewart, J. Low Temp. Phys. 105, 37 (1996).

13. K. Gloos, Phys. Rev. Lett. 85, 5257 (2000).

14. N.W. Ashcroft and N.D. Mermin, Solid State Physic, Thomson Learning, (1976), ch. 2.

15. D.R. Hamann, Phys. Rev. 158, 570 (1967).

16. K. Gloos, Czechoslovak. J. Phys. 46 Suppl. S4, 2055 (1996).

17. K. Gloos and F. Martin, Z. Phys. B-Condensed Matter 99, 321 (1996).

18. G.E. Blonder, M. Tinkham, and T.M. Klapwijk, Phys. Rev. B25, 4515 (1982)

19. L. Vitali, R. Ohmann, S. Stepanow, P. Gambardella, K. Tao, R. Huang, V.S. Stepanyuk, P. Bruno, and K. Kern, Phys. Rev. Lett. 101, 216802 (2008).

20. Y. Meir, N.S. Wingreen, and P.A. Lee, Phys. Rev. Lett. 70, 2601 (1993).

21. D. Goldhaber-Gordon, Hadas Shtrikman, D. Mahalu, D. Abusch-Magder, U. Meirav, and M.A. Kastner, Nature 391, 156 (1998)

22. S.M. Cronenwett, T.H. Oosterkamp, and L.P. Kouwenhoven, Science 281, 540 (1998).

23. J.P. Bird and Y. Ochiai, Science 303, 1621 (2004).

24. T. Rejec and Y. Meir, Nature 442, 900 (2006).

25. A.M. Duif, A.G. M. Jansen, and P. Wyder, J. Phys. Cond. Matter 1, 3157 (1987).

26. K. Kokko, private communication.

27. M.R. Calvo, J. Fernández-Rossier, J.J. Palacios, D. Jacob, D. Natelson, and C. Untiedt, Nature 458, 1150 (2009). 